\title{
Radiotherapy with neoadjuvant chemotherapy versus concurrent chemoradiotherapy for ascending-type nasopharyngeal carcinoma: a retrospective comparison of toxicity and prognosis
}

Ji-Jin Yao ${ }^{1,2 \dagger}$, Xiao-Li Yu ${ }^{3 \dagger}$, Fan Zhang ${ }^{2 \dagger}$, Wang-Jian Zhang ${ }^{4}$, Guan-Qun Zhou ${ }^{1}$, Ling-Long Tang ${ }^{1}$, Yan-Ping Mao ${ }^{1}$, Lei Chen ${ }^{1}$, Jun $\mathrm{Ma}^{1}$ and Ying Sun ${ }^{*}$

\begin{abstract}
Background: In the era of intensity-modulated radiotherapy (IMRT), the role of neoadjuvant chemotherapy (NACT) in treating ascending-type nasopharyngeal carcinoma (NPC) is under-evaluated. This study was to compare the efficacy of NACT followed by IMRT (NACT + RT) with the efficacy of concurrent chemoradiotherapy (CCRT) on ascending-type NPC.

Methods: Clinical data of 214 patients with ascending-type NPC treated with NACT + RT or CCRT between December 2009 and July 2011 were analyzed. Of the 214 patients, 98 were treated with NACT followed by IMRT, and 116 were treated with CCRT. The survival rates were assessed using Kaplan-Meier analysis, and the survival curves were compared using a log-rank test.

Results: The 4-year overall survival, locoregional failure-free survival, distant failure-free survival, and failure-free survival rates were not significantly different between the two groups (all $P>0.05$ ). However, patients in the CCRT group exhibited more severe acute adverse events than did patients in the NACT + RT group during radiotherapy, including leukopenia (30.2\% vs. $15.3 \%, P=0.016$ ), neutropenia ( $25.9 \%$ vs. $11.2 \%, P=0.011)$, and mucositis (57.8\% vs. $40.8 \%$, $P=0.028)$. After radiotherapy, patients in the CCRT group exhibited significantly higher rates of xerostomia ( $21.6 \% \mathrm{vs}$. $10.2 \%, P=0.041)$ and hearing loss $(17.2 \%$ vs. $6.1 \%, P=0.023)$.
\end{abstract}

Conclusions: The treatment outcomes of the NACT + RT and CCRT groups were similar; however, CCRT led to higher rates of acute and late toxicities. NACT + RT may therefore be a better treatment strategy for ascending-type NPC.

Keywords: Nasopharyngeal carcinoma, Ascending-type, Intensity-modulated radiotherapy, Neoadjuvant chemotherapy, Concurrent chemoradiotherapy

\section{Background}

Nasopharyngeal carcinoma (NPC) is a common disease in South China [1]. In contrast to patients with

\footnotetext{
*Correspondence: sunying@sysucc.org.cn

${ }^{\dagger} \mathrm{Ji}$-Jin Yao, Xiao-Li Yu and Fan Zhang contributed equally to this work

${ }^{1}$ Department of Radiation Oncology, State Key Laboratory of Oncology in South China, Collaborative Innovation Center for Cancer Medicine,

Sun Yat-sen University Cancer Center, 651 Dongfeng Road East,

Guangzhou 510060, Guangdong, P. R. China

Full list of author information is available at the end of the article
}

early-stage NPC, who have 5-year overall survival (OS) rates of up to $95 \%$ [2-4], the 5-year OS rate declines to $41 \%-63 \%$ in patients with advanced-stage disease $[5,6]$. Thus, there is a clear need to improve the treatment outcome for advanced-stage NPC.

Previous publications $[7,8]$ and meta-analyses $[9,10]$ have reported that concurrent chemoradiotherapy (CCRT) provides greater survival benefits than neoadjuvant chemotherapy (NACT) followed by radiotherapy. Additionally, a clinical trial [11] and a meta-analysis [12] found that 
NACT followed by CCRT was well tolerated and provided good outcomes, whereas others [13-15] question the value of concurrent chemotherapy for intensity-modulated radiotherapy (IMRT)-treated patients with locoregionally advanced NPC. These discrepancies may be partly due to the biological heterogeneity of NPC cases in the study populations. As reported by Wee et al. [16], NPC patients with predominantly advanced local disease (advanced $\mathrm{T}$ stage) and early-stage cervical lymph node involvement (early $\mathrm{N}$ stage) are classified as having the ascending type of the disease, who usually experiences local failure, whereas those with early-stage local disease (early $\mathrm{T}$ stage) and advanced lymph node metastases (advanced $\mathrm{N}$ stage) are classified as having the descending type, for whom distant failure is more common than local failure. These two types of NPC can exhibit distinct clinical-biological behaviors [17].

These previous studies did not take tumor heterogeneity into account, and differences in the numbers of cases of the ascending and descending types in the study populations could have affected the conclusions. Therefore, we included only ascending-type NPC cases in our study to avoid the effect of such tumor heterogeneity. The objective of the present study was to assess the efficacy of NACT followed by IMRT (NACT + RT) versus concurrent chemotherapy with IMRT (CCRT) on ascendingtype NPC.

\section{Patients and methods}

\section{Patient selection}

Consecutive patients with newly diagnosed, histologically proven, non-distant metastatic, ascending-type NPC that was treated with IMRT between December 2009 and July 2011 at the Sun Yat-sen University Cancer Center (Guangzhou, China) were selected. The need for written consent was waived, whereas oral consent was obtained from the patients via telephone, as documented by telephone recording. The use of oral consent was approved by the institutional review board.

All patients completed a pretreatment evaluation, which included physical examination, chest radiography, nasopharyngeal and neck magnetic resonance imaging (MRI), abdominal sonography, and a whole-body bone scan. Patients were restaged by two radiation oncologists specializing in head and neck cancer according to the 7th edition of the Union for International Cancer Control/American Joint Committee on Cancer (UICC/ AJCC) staging system, with disagreements resolved by consensus.

\section{Radiotherapy}

The primary tumor and the upper neck area above the caudal edge of the cricoid cartilage were treated with IMRT. Target volumes were delineated according to our institutional treatment protocol [18], in agreement with the International Commission on Radiation Units and Measurements (ICRU) Reports 62 [19] and 83 [20]. The gross tumor volume (GTV) including primary nasopharyngeal tumor (GTVp) and involved lymph nodes (GTVnd) was delineated on the basis of physical examination and MRI findings. Gross disease at primary site together with enlarged retropharyngeal lymph nodes was designated GTVp; clinically-involved cervical lymph nodes, GTVnd. Two clinical target volumes (CTVs) were delineated according to the GTV: CTV1, high-risk regions encompassing GTVp plus 5-10 $\mathrm{mm}$, including the entire nasopharyngeal mucosa and $5-\mathrm{mm}$ submucosal region; and CTV2, low-risk regions containing CTV1 plus $5-10 \mathrm{~mm}$, encompassing sites of microscopic extension and lymphatic regions. The planning target volumes (PTVs), termed PTVp, PTV1, PTV2, and PTVnd, were constructed by expanding the GTVp, CTV1, CTV2, and CTVnd, respectively, by $3 \mathrm{~mm}$; a $3 \mathrm{~mm}$ margin was added to the brainstem and spinal cord to generate planning organ at risk volume (PRV).

The prescribed doses to PTVp, PTVnd, PTV1, and PTV2 were 66-72, 64-70, 60-63, and 54-56 Gy, respectively, in 28-33 fractions (66-70 Gy to PTVp for T1 NPC and 68-72 Gy for T2-4 NPC; 68-70 Gy to clinically-involved nodes $>1 \mathrm{~cm}$ in diameter and 64-68 Gy to nodes $\leq 1 \mathrm{~cm}$ in diameter) [21]. The dose constraints for organs at risk (OARs) and PRVs were as described in the Radiation Therapy Oncology Group (RTOG)-0225 trial [22]. All patients were treated following a routine schedule (one fraction daily for 5 days per week).

Boost treatment was offered for selected patients at the attending physician's discretion, usually in cases of bulky or suspected residual disease. This additional radiation was delivered by high-dose-rate intracavitary brachytherapy or external beam radiation.

\section{Chemotherapy}

During the study period, institutional guidelines recommended neoadjuvant or adjuvant chemotherapy and/ or CCRT for stage III to IVA-B NPC. For these patients, NACT was given when it was considered advantageous to reduce bulky tumors or when the waiting time for radiotherapy was considered to be longer than acceptable. The NACT consisted of cisplatin (80-100 mg/ $\mathrm{m}^{2}$, intravenous infusion on day 1) plus 5 -fluorouracil (800-1000 mg/m², 120-h continuous intravenous infusion) or cisplatin $\left(80-100 \mathrm{mg} / \mathrm{m}^{2}\right.$, intravenous infusion on day 1) plus docetaxel $\left(60-80 \mathrm{mg} / \mathrm{m}^{2}\right.$, intravenous infusion on day 1 ) every 3 weeks for three cycles. Concurrent chemotherapy consisted of cisplatin $\left(80-100 \mathrm{mg} / \mathrm{m}^{2}\right.$, intravenous infusion on day 1 ) given in weeks 1,4 , and 
7 of radiotherapy or cisplatin $\left(30-45 \mathrm{mg} / \mathrm{m}^{2}\right.$, intravenous infusion on day 1) given weekly during radiotherapy. Deviations from the institutional guidelines were due to organ dysfunction (suggesting intolerance to the chemotherapy) or patient refusal.

\section{Patient assessment and follow-up}

Our primary study endpoint was OS, defined as the duration from the date of treatment initiation to the date of cancer-related death or the last follow-up. The secondary endpoints included locoregional failure-free survival (LRFFS), defined as the duration from the date of treatment initiation to the date of the first relapse in the nasopharyngeal and/or cervical region or the last follow-up; distant failure-free survival (DFFS), defined as the duration from the date of treatment initiation to the date of the first distant metastasis or the last follow-up; and failure-free survival (FFS), defined as the duration from the date of treatment initiation to the date of disease progression (local/regional recurrence or distant metastasis), death from any cause, or the last follow-up. Acute and late toxicities were documented according to the Common Terminology Criteria for Adverse Events (CTCAE) version 3.0 and/or the Radiation Morbidity Scoring Criteria of the RTOG.

The last follow-up visit was in November 2015. Patients were assessed at least every 3 months in the first 3 years and every 6 months thereafter. Routine follow-up included complete head and neck examination, nasopharyngoscopy, hematology and biochemistry profiles, chest radiography, and abdominal sonography. Follow-up neck and/or nasopharyngeal MRI was performed every 6-12 months, especially for patients with suspected tumor recurrence or radiotherapy-induced complications.

\section{Statistical analysis}

To compare clinicopathologic features between the NACT + RT and CCRT groups, a Chi square $\left(x^{2}\right)$ test was used for categorical variables, and a Kruskal-Wallis rank-sum test was used for continuous variables. The Kaplan-Meier method was used to estimate the rates of OS, LRFFS, DFFS, and FFS. The log-rank test was used to compare the survival rates between the NACT + RT and CCRT groups. All statistical tests were two-sided, and $P<0.05$ was considered statistically significant. Statistical analyses were performed with $\mathrm{R}$ version 3.1.2.

\section{Results}

\section{Patient characteristics}

Between December 2009 and July 2011, 239 consecutive patients with ascending-type NPC were treated at our center. Of the 239 patients, 25 were excluded: 6 received adjuvant chemotherapy, 8 received both NACT and concurrent chemotherapy, and 11 received radiotherapy alone. Therefore, a total of 214 patients were included in the study: 98 were treated with NACT + RT, and 116 were treated with CCRT.

All patients had biopsy-proven NPC and an adequate performance status for radical treatment (Karnofsky performance score $\geq 70$ ). Clinicopathologic features were well balanced between the two groups (Table 1), and there were no significant differences in the radiotherapy dose or duration. The median follow-up period was 46.8 months (range 8.9-70.3 months).

\section{Treatment compliance}

All patients completed the planned course of IMRT. Six patients received boost treatment after the planned course of IMRT due to the presence of gross residual disease (i.e., $<100 \%$ resolution of the primary disease), as observed on follow-up MRI, or nasopharyngoscopy. Of these patients, four were treated with a brachytherapy boost (12-16 Gy at 3-4 Gy per daily fraction), and two

Table 1 Clinicopathologic features of the neoadjuvant chemotherapy plus radiotherapy (NACT + RT) group and the concurrent chemoradiotherapy (CCRT) group of patients with ascending-type nasopharyngeal carcinoma (NPC)

\begin{tabular}{llll}
\hline Variable & NACT + RT group & CCRT group & P value \\
\hline Total & 98 & 116 & \\
Age (years) & & & 0.367 \\
$\quad$ Median & 44 & 45 & \\
Range & $17-70$ & $16-70$ & \\
Gender [cases (\%)] & & & 0.179 \\
Male & $84(85.7)$ & $90(77.6)$ & \\
Female & $14(14.3)$ & $26(22.4)$ & \\
Histology [cases (\%)] & & & 0.795 \\
WHO II & $5(5.1)$ & $8(6.9)$ & \\
WHO III & $93(94.9)$ & $108(93.1)$ & \\
T stage [cases (\%)] & & & \\
T3 & $43(43.9)$ & $64(55.2)$ & \\
T4 & $55(56.1)$ & $52(44.8)$ & \\
N stage [cases (\%)] & & & \\
N0 & $34(34.7)$ & $47(40.5)$ & \\
N1 & $64(65.3)$ & $69(59.5)$ & \\
Total radiation dose (Gy) & & & \\
Median & 70 & 6.721 \\
Range & $66-72$ & $66-72$ & \\
Overall duration of radiotherapy (days) & 46 & 47 & \\
Median & $40-49$ & & \\
Range & & & \\
\hline
\end{tabular}

WHO World Health Organization

a According to the Union for International Cancer Control/American Joint Committee on Cancer staging system, 7th edition 
were treated with external beam irradiation (11 Gy at 2.2 Gy per daily fraction).

In the NACT + RT group, 42 patients were treated with a PF regimen (cisplatin and 5-fluorouracil), and 56 patients were treated with a TP regimen (cisplatin and docetaxel). Six patients (6.1\%) in the NACT + RT group completed only one cycle of NACT: 1 developed febrile neutropenia, 2 exhibited impaired liver function, and 3 refused further chemotherapy. The other 92 patients $(93.9 \%)$ in the NACT + RT group completed 2-3 cycles of NACT. In the CCRT group, 58 patients (50.0\%) were treated with cisplatin every 3 weeks during radiotherapy, and the other 58 (50.0\%) were treated with weekly cisplatin during radiotherapy. Of those who were treated with cisplatin every 3 weeks, 44 (75.9\%) completed two cycles, and 14 (24.1\%) completed three cycles. Of those who were treated with weekly cisplatin, $52(89.6 \%)$ completed at least four cycles. The median doses of cisplatin were $185 \mathrm{mg} /$ $\mathrm{m}^{2}$ for the NACT + RT group and $200 \mathrm{mg} / \mathrm{m}^{2}$ for the CCRT group.

\section{Acute and late toxicities}

In the NACT + RT group, among the severe to lifethreatening (grade 3-4) hematologic adverse events that were observed during NACT, neutropenia was the most common (24 patients; 24.5\%), and the most common non-hematologic adverse event was nausea/vomiting (6 patients; $6.1 \%$ ). Other grade 3-4 acute adverse events included leukopenia, thrombocytopenia, hepatotoxicity, and nephrotoxicity (Table 2).

Acute toxicities during radiotherapy were well tolerated by both groups. During radiotherapy, the rates of grade 3-4 adverse events were higher in the CCRT group than in the NACT + RT group (mucositis: $57.8 \%$ vs. $40.8 \%, P=0.028$; leukopenia: $30.2 \%$ vs. $15.3 \%, P=0.016$; and neutropenia: $25.9 \%$ vs. $11.2 \%, P=0.011$ ) (Table 2).

Common late adverse events included xerostomia, hearing loss, neuropathy, and neck tissue damage. The rates of grade 3-4 xerostomia and hearing loss were significantly lower in the NACT + RT group than in the CCRT group $(10.2 \%$ vs. $21.6 \%, P=0.041 ; 6.1 \%$ vs. $17.2 \%$, $P=0.023)$. The rates of all other adverse events were

Table 2 Acute and late adverse events in the NACT + RT and CCRT groups

\begin{tabular}{|c|c|c|c|c|c|}
\hline \multirow[t]{2}{*}{ Adverse event } & \multicolumn{2}{|c|}{ NACT + RT group [cases (\%)] } & \multicolumn{2}{|c|}{ CCRT group [cases (\%)] } & \multirow{2}{*}{$\begin{array}{l}P \\
\text { value }^{\dagger}\end{array}$} \\
\hline & Grade 1-4 & Grade 3-4 & Grade 1-4 & Grade 3-4 & \\
\hline \multicolumn{6}{|l|}{ Neoadjuvant phase } \\
\hline Neutropenia & $61(62.2)$ & $24(24.5)$ & NA & NA & NA \\
\hline Leukopenia & $54(55.1)$ & $20(20.4)$ & NA & NA & NA \\
\hline Thrombocytopenia & $11(11.2)$ & $4(4.1)$ & NA & NA & NA \\
\hline Nausea/vomiting & $47(48.0)$ & $6(6.1)$ & NA & NA & NA \\
\hline Hepatotoxicity & $53(54.1)$ & $3(3.1)$ & NA & NA & NA \\
\hline Nephrotoxicity & $37(37.8)$ & $1(1.0)$ & NA & NA & NA \\
\hline \multicolumn{6}{|l|}{ Irradiation phase } \\
\hline Mucositis & $98(100)$ & $40(40.8)$ & $116(100)$ & $67(57.8)$ & 0.028 \\
\hline Xerostomia & $98(100)$ & $24(24.5)$ & $116(100)$ & $35(30.2)$ & 0.439 \\
\hline Skin & $98(100)$ & $11(11.2)$ & $116(100)$ & $19(16.4)$ & 0.376 \\
\hline Nausea/vomiting & $41(41.8)$ & $7(7.1)$ & $68(58.6)$ & $10(8.6)$ & 0.885 \\
\hline Leukopenia & $43(43.9)$ & $15(15.3)$ & $71(61.2)$ & $35(30.2)$ & 0.016 \\
\hline Neutropenia & $36(36.7)$ & $11(11.2)$ & $75(64.7)$ & $30(25.9)$ & 0.011 \\
\hline Thrombocytopenia & $15(15.3)$ & $3(3.1)$ & $24(20.7)$ & $7(6.0)$ & 0.157 \\
\hline Hepatotoxicity & $28(28.6)$ & $1(1.0)$ & $40(34.5)$ & $2(1.7)$ & 0.864 \\
\hline Nephrotoxicity & $22(22.4)$ & $0(0)$ & $32(27.6)$ & $0(0)$ & 0.789 \\
\hline \multicolumn{6}{|l|}{ Post-irradiation phase } \\
\hline Xerostomia & $98(100)$ & $10(10.2)$ & $116(100)$ & $25(21.6)$ & 0.041 \\
\hline Hearing loss & $34(34.7)$ & $6(6.1)$ & $69(59.5)$ & $20(17.2)$ & 0.023 \\
\hline Neuropathy & $24(24.5)$ & $2(2.0)$ & 37 (31.9) & $1(0.9)$ & 0.761 \\
\hline Neck tissue damage & $9(9.2)$ & $0(0)$ & 15 (12.9) & $0(0)$ & 0.867 \\
\hline Dysphagia & $1(1.0)$ & $0(0)$ & $2(1.7)$ & $0(0)$ & 0.998 \\
\hline
\end{tabular}

Comparison of the incidence of grade 3-4 events between the two groups

$N A$ not applicable

${ }^{\dagger} P$ values were calculated using a $C$ hi square test 
not significantly different between the two groups (all $P>0.05)$ (Table 2).

\section{Treatment outcomes}

The 4-year OS rates were $92.3 \%$ in the NACT + RT group and $82.1 \%$ in the CCRT group (Fig. 1a). The two groups had a similar risk of death $(\mathrm{HR}=0.41 ; 95 \% \mathrm{CI}$ $0.16-1.12 ; P=0.072$ ). The 4 -year LRFFS rates were $87.2 \%$ in the NACT + RT group and $79.5 \%$ in the CCRT group (Fig. 1b), and the HR for the treatment effect was 0.66 (95\% CI $0.32-1.38 ; P=0.268)$. Fourteen patients in the $\mathrm{NACT}+\mathrm{RT}$ group and 25 in the CCRT group developed locoregional failure. The 4-year DFFS rates were $84.7 \%$ in the NACT + RT group and $81.4 \%$ in the CCRT group (Fig. 1c), and the HR for the treatment effect was 0.77 (95\% CI $0.33-1.78 ; P=0.539$ ). Thirteen patients in the NACT + RT group and 23 in the CCRT group developed distant metastases. The 4-year FFS rates were 76.3\% in the NACT + RT group and $73.2 \%$ in the CCRT group (Fig. 1d), and the HR for the treatment effect was 0.70 (95\% CI 0.37-1.36; $P=0.293$ ). Twenty-four patients in the NACT + RT group and 32 in the CCRT group developed locoregional failure and/or distant metastases.

\section{Discussion}

In the current study, we retrospectively compared the efficacy of NACT + RT with the efficacy of CCRT on ascending-type NPC. The results showed that $\mathrm{NACT}+\mathrm{RT}$ for ascending-type NPC provided a favorable outcome in terms of 4-year OS, LRFFS, DFFS, and FFS rates. In consistent with our results, Lin et al. [15] and Qiu et al. [23] investigated patients with locoregionally advanced NPC who were treated with IMRT after NACT and demonstrated that NACT + RT produced a superb outcome in terms of OS, LRFFS, DFFS, and FFS rates in these patients. Our results further suggested that CCRT offered no significant survival benefit when compared with NACT + RT.

The treatment outcome with the application of IMRT has been demonstrated in terms of locoregional control in NPC patients [24]. The 3-year LRFFS and OS rates of NPC patients were $90 \%-95 \%$ and $80 \%-85 \%$, respectively
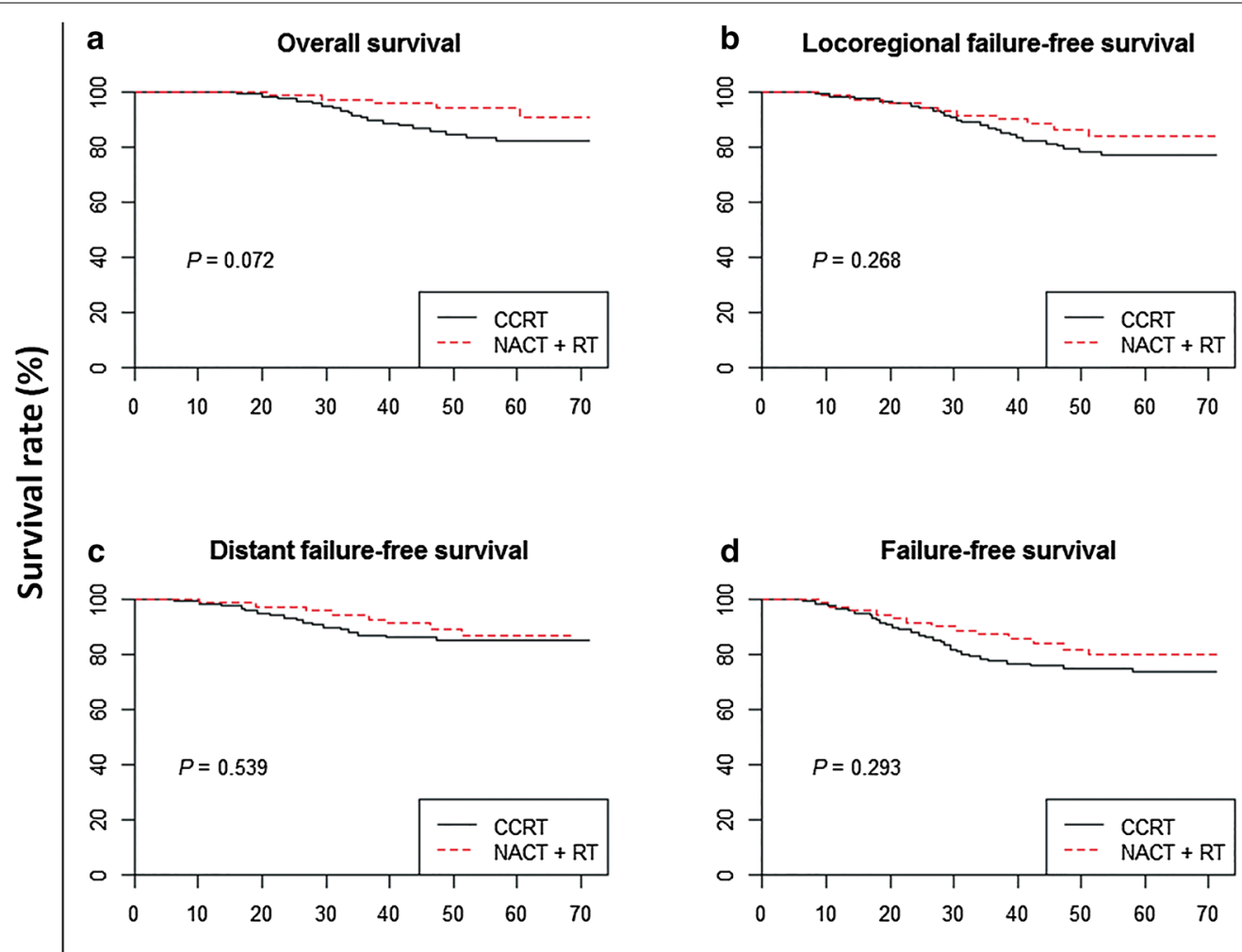

\section{Time after treatment initiation (months)}

Fig. 1 Kaplan-Meier estimates of the survival of patients with the ascending type of nasopharyngeal carcinoma (NPC) treated with neoadjuvant chemotherapy plus subsequent radiotherapy (NACT + RT) or with concurrent chemoradiotherapy (CCRT). a Overall survival; b locoregional failurefree survival; c distant failure-free survival; $\mathbf{d}$ failure-free survival 
[25-28]. Improved locoregional control was anticipated with improved dose coverage of GTV and CTV. Therefore, the effect of chemotherapy on locoregional control in NPC patients treated with IMRT remained uncertain. Our results demonstrated that for patients with ascending-type NPC, CCRT provided no significant improvement in terms of LRFFS when compared with $\mathrm{NACT}+\mathrm{RT}$; the 4-year LRFFS rate was, in fact, higher in the NACT + RT group ( $87.2 \%$ vs. $79.5 \%)$, though the difference was not significant $(P=0.268)$. Lai et al. [29] found that LRFFS was significantly longer for patients who underwent IMRT than for patients who underwent two-dimensional conventional radiotherapy (2D-CRT). Compared with 2D-CRT, IMRT generates more conformal dose coverage in the target volume and therefore results in better local control. Thus, it is possible that the improved local control rates obtained with IMRT eliminated the contribution of concurrent chemotherapy to LRFFS. Furthermore, significant NACT-induced shrinkage of the tumor prior to radiotherapy increases the margin of safety between the tumor and irradiation volumes, as reported by Teo et al. [30], which could reduce the locoregional recurrence rate in patients with locoregionally advanced NPC.

Although the main advantage of NACT is reduction of distant metastases [31], the NACT + RT group did not exhibit a significantly lower rate of distant metastases compared with the CCRT group in the present study. This observation may be interpreted as follows. First, the tendency in this study was to give NACT to patients when it was considered advantageous to reduce bulky tumors or when the waiting time for radiotherapy was considered to be longer than acceptable. For those patients, tumor volumes were relatively large despite the same $\mathrm{T}$ stage. However, previous studies have revealed that the primary tumor volume is closely related to disease progression in NPC [32, 33]. Guo et al. [34] demonstrated that a large tumor volume was predictive of a poor prognosis and was associated with distant metastasis in NPC patients treated with IMRT. Second, this analysis may have been affected by the smaller size of the NACT + RT group compared with that of the CCRT group [98 (45.8\%) vs. $116(54.2 \%)]$.

Clearly, treatment-induced adverse events could limit efficacy in patients with locoregionally advanced NPC. In this study, specific adverse events were associated with each of the two treatment protocols. Compared with the NACT + RT group, the CCRT group suffered significantly more acute adverse events such as neutropenia, leukopenia, and mucositis during radiotherapy. These findings are consistent with those reported in previous studies that used similar irradiation techniques [35, 36]. There was also a significant difference between our two groups in terms of late adverse events: patients in the CCRT group exhibited significantly more xerostomia and hearing loss than patients in the NACT + RT group. Two underlying reasons for these findings could be as follows: first, concurrent chemotherapy may increase the sensitivity of normal tissue to radiotherapy-related injury, and second, the total radiation dose to nearby organs may be reduced after the primary tumor has been shrunken by NACT.

Compared with the CCRT group in the present study, Kong et al. [37] showed lower rates of severe irradiation-related mucositis and xerostomia in advanced NPC patients treated with NACT + CCRT. In the current study, only the patients with ascending-type NPC, predominantly advanced local disease (T3-4) with early-stage cervical lymph node involvement (N0-1), were selected, whereas Kong et al. [37] did not take into account the variability of the tumor itself. With ascending-type NPC, patients have high radiation doses to OARs due to the advanced $\mathrm{T}$ stage and large target volume. For example, despite the same stage (stage IV) and the same treatment for patients with T1N3 and T4N0 NPC, the adverse events might be markedly different.

\section{Conclusions}

Compared with NACT + RT, CCRT did not significantly increase the 4-year OS, LRFFS, and FFS rates of patients with ascending-type NPC, and CCRT was associated with higher rates of severe adverse events. These results suggest that NACT + RT may be a more suitable treatment strategy for ascending-type NPC. However, our study was limited by the small sample size and the use of retrospective analysis. A prospective randomized trial will be needed to confirm the findings.

\section{Authors' contributions}

YJJ, YXL, and ZF collected data and drafted the manuscript. ZWJ and ZGQ performed the statistical analyses. TLL, MYP, CL, and MJ participated in the design of the study. SY conceived of the study and participated in its design. All authors read and approved the final manuscript.

\section{Author details}

${ }^{1}$ Department of Radiation Oncology, State Key Laboratory of Oncology in South China, Collaborative Innovation Center for Cancer Medicine, Sun Yatsen University Cancer Center, 651 Dongfeng Road East, Guangzhou 510060, Guangdong, P. R. China. ${ }^{2}$ Department of Radiation Oncology, The Fifth Affiliated Hospital of Sun Yat-sen University, Zhuhai 519001, Guangdong, P. R. China. ${ }^{3}$ Department of Radiation Oncology, Sun Yat-sen Memorial Hospital, Sun Yat-sen University, Guangzhou 510120, Guangdong, P. R. China. ${ }^{4}$ Department of Medical Statistics and Epidemiology \& Health Information Research Center \& Guangdong Key Laboratory of Medicine, School of Public Health, Sun Yat-sen University, Guangzhou 510080, Guangdong, P. R. China.

\section{Acknowledgements}

This work was supported by grants from the Science and Technology Project of Guangzhou City, China (No. 14570006), the National Natural Science Foundation of China (Nos. 81372409, 81402532), and the Sun Yat-sen University Clinical Research 5010 Program (No. 2012011). The funders had no role in 
study design, data collection and analysis, decisions regarding publication, or preparation of the manuscript.

\section{Competing interests}

The authors declare that they have no competing interests.

Received: 6 March 2016 Accepted: 21 June 2016

Published online: 06 March 2017

\section{References}

1. Wei KR, Zheng RS, Zhang SW, Liang ZH, Ou ZX, Chen WQ. Nasopharyngeal carcinoma incidence and mortality in China in 2010. Chin J Cancer. 2014;33(8):381-7. doi:10.5732/cjc.014.10086.

2. Cao XP, Lu TX, Ye WJ, Cui NJ. Prospective study on long-term efficacy of external plus intracavitary radiotherapy on stage I-II nasopharyngeal carcinoma. Ai Zheng. 2007;26:204-7.

3. Leung TW, Tung SY, Sze WK, Wong FC, Yuen KK, Lui CM, et al. Treatment results of 1070 patients with nasopharyngeal carcinoma: an analysis of survival and failure patterns. Head Neck. 2005;27:555-65.

4. Xiao WW, Han F, Lu TX, Chen CY, Huang Y, Zhao C. Treatment outcomes after radiotherapy alone for patients with early-stage nasopharyngeal carcinoma. Int J Radiat Oncol Biol Phys. 2009;74:1070-6.

5. El-Sherbieny E, Rashwan H, Lubis SH, Choi VJ. Prognostic factors in patients with nasopharyngeal carcinoma treated in Hospital Kuala Lumpur. Asian Pac J Cancer Prev. 2011;12(7):1739-43.

6. Tao CJ, Liu X, Tang LL, Mao YP, Chen L, Li WF, et al. Prognostic scoring system for locoregional control among the patients with nasopharyngeal carcinoma treated by intensity-modulated radiotherapy. Chin J Cancer. 2013;32(9):494-501. doi:10.5732/cjc.013.10121.

7. Lin JC, Jan JS, Hsu CY, Liang WM, Jiang RS, Wang WY. Phase III study of concurrent chemoradiotherapy versus radiotherapy alone for advanced nasopharyngeal carcinoma: positive effect on overall and progressionfree survival. J Clin Oncol. 2003;21:631-7.

8. Chan AT, Leung SF, Ngan RK, Teo PM, Lau WH, Kwan WH, et al. Overall survival after concurrent cisplatin-radiotherapy compared with radiotherapy alone in locoregionally advanced nasopharyngeal carcinoma. J Natl Cancer Inst. 2005;97:536-9.

9. Langendijk JA, Leemans CR, Buter J, Berkhof J, Slotman BJ. The additional value of chemotherapy to radiotherapy in locally advanced nasopharyngeal carcinoma: a meta-analysis of the published literature. J Clin Oncol. 2004;22:4604-12.

10. Baujat B, Audry H, Bourhis J, Chan AT, Onat H, Chua DT, et al. Chemotherapy in locally advanced nasopharyngeal carcinoma: an individual patient data meta-analysis of eight randomized trials and 1753 patients. Int J Radiat Oncol Biol Phys. 2006;64:47-56.

11. Hui EP, Ma BB, Leung SF, King AD, Mo F, Kam MK, et al. Randomized phase II trial of concurrent cisplatinradiotherapy with or without neoadjuvant docetaxel and cisplatin in advanced nasopharyngeal carcinoma. J Clin Oncol. 2009;27:242-9.

12. Chen YP, Guo R, Liu N, Liu X, Mao YP, Tang LL, et al. Efficacy of the additional neoadjuvant chemotherapy to concurrent chemoradiotherapy for patients with locoregionally advanced nasopharyngeal carcinoma: a Bayesian network meta-analysis of randomized controlled trials. J Cancer. 2015;6(9):883-92.

13. Ma J, Mai HQ, Hong MH, Min HQ, Mao ZD, Cui NJ, et al. Results of a prospective randomized trial comparing neoadjuvant chemotherapy plus radiotherapy with radiotherapy alone in patients with locoregionally advanced nasopharyngeal carcinoma. J Clin Oncol. 2001;19:1350-7.

14. Hareyama M, Sakata K, Shirato H, Nishioka T, Nishio M, Suzuki K, et al. A prospective, randomized trial comparing neoadjuvant chemotherapy with radiotherapy alone in patients with advanced nasopharyngeal carcinoma. Cancer. 2002;94:2217-23.

15. Lin S, Lu JJ, Han L, Chen Q, Pan J. Sequential chemotherapy and intensity modulated radiation therapy in the management of locoregionally advanced nasopharyngeal carcinoma: experience of 370 consecutive cases. BMC Cancer. 2010;10:39

16. Wee J, Tan EH, Tai BC, Wong HB, Leong SS, Tan T, et al. Randomized trial of radiotherapy versus concurrent chemoradiotherapy followed by adjuvant chemotherapy in patients with American Joint Committee on Cancer/ International Union against cancer stage III and IV nasopharyngeal cancer of the endemic variety. J Clin Oncol. 2005;23(27):6730-8.

17. Sun P, Chen C, Chen XL, Cheng YK, Zeng L, Zeng ZJ, et al. Proposal of a clinical typing system and generation of a prognostic model in patients with nasopharyngeal carcinoma from Southern China. J BUON. 2014;19(2):474-83.

18. Zhao C, Han F, Lu LX, Huang SM, Lin CG, Deng XW, et al. Intensity modulated radiotherapy for local-regional advanced nasopharyngeal carcinoma. Ai Zheng. 2004;23(11 Suppl):1532-7.

19. ICRU report. Prescribing, recording, and reporting photon beam therapy. vol. 62. Maryland: International Commission on Radiation Units and Measurements; 1999.

20. ICRU Report. Prescribing, recording, and reporting photon-beam intensity-modulated radiation therapy (IMRT). vol. 83. Maryland: International Commission on Radiation Units and Measurements; 2010.

21. Su SF, Han F, Zhao C, Chen CY, Xiao WW, Li JX, et al. Long-term outcomes of early-stage nasopharyngeal carcinoma patients treated with intensity-modulated radiotherapy alone. Int J Radiat Oncol Biol Phys. 2012;82(1):327-33. doi:10.1016/j.jijobp.2010.09.011.

22. Lee N, Harris J, Garden AS, Straube W, Glisson B, Xia P, et al. Intensity-modulated radiation therapy with or without chemotherapy for nasopharyngeal carcinoma: radiation therapy oncology group phase II trial 0225. J Clin Oncol. 2009:27(22):3684-90.

23. Qiu WZ, Huang PY, Shi JL, Xia HQ, Zhao C, Cao KJ. Neoadjuvant chemotherapy plus intensity-modulated radiotherapy versus concurrent chemoradiotherapy plus adjuvant chemotherapy for the treatment of locoregionally advanced nasopharyngeal carcinoma: a retrospective controlled study. Chin J Cancer. 2016;35(1):2. doi:10.1186/ s40880-015-0076-9.

24. Zhang MX, Li J, Shen GP, Zou X, Xu JJ, Jiang R, et al. Intensity-modulated radiotherapy prolongs the survival of patients with nasopharyngeal carcinoma compared with conventional two-dimensional radiotherapy: a 10-year experience with a large cohort and long follow-up. Eur I Cancer. 2015;51(17):2587-95. doi:10.1016/j.ejca.2015.08.006.

25. Lee N, Xia P, Quivey JM, Sultanem K, Poon I, Akazawa C, et al. Intensitymodulated radiotherapy in the treatment of nasopharyngeal carcinoma: an update of the UCSF experience. Int J Radiat Oncol Biol Phys. 2002;53(1):12-22.

26. Kam MK, Teo PM, Chau RM, Cheung KY, Choi PH, Kwan WH, et al. Treatment of nasopharyngeal carcinoma with intensity-modulated radiotherapy: the Hong Kong experience. Int J Radiat Oncol Biol Phys. 2004;60(5):1440-50.

27. Tham IW, Hee SW, Yeo RM, Salleh PB, Lee J, Tan TW, et al. Treatment of nasopharyngeal carcinoma using intensity-modulated radiotherapyThe National Cancer Centre Singapore experience. Int J Radiat Oncol Biol Phys. 2009;75:1481-6.

28. Lin S, Pan J, Han L, Zhang X, Liao X, Lu JJ. Nasopharyngeal carcinoma treated with reduced-volume intensity-modulated radiation therapy: report on the 3-year outcome of a prospective series. Int J Radiat Oncol Biol Phys. 2009;75(4):1071-8. doi:10.1016/j.jijobp.2008.12.015.

29. Lai SZ, Li WF, Chen L, Luo W, Chen YY, Liu LZ, et al. How does intensitymodulated radiotherapy versus conventional two-dimensional radiotherapy influence the treatment results in nasopharyngeal carcinoma patients? Int J Radiat Oncol Biol Phys. 2011:80:661-8.

30. Teo PM, Chan AT, Lee WY, Leung TW, Johnson PJ. Enhancement of local control in locally advanced node-positive nasopharyngeal carcinoma by adjunctive chemotherapy. Int J Radiat Oncol Biol Phys. 1999;43:261-71.

31. OuYang PY, Xie C, Mao YP, Zhang Y, Liang XX, Su Z, et al. Significant efficacies of neoadjuvant and adjuvant chemotherapy for nasopharyngeal carcinoma by meta-analysis of published literature-based randomized, controlled trials. Ann Oncol. 2013;24:2136-46.

32. Chua DT, Sham JS, Kwong DL, Tai KS, Wu PM, Lo M, et al. Volumetric analysis of tumor extent in nasopharyngeal carcinoma and correlation with treatment outcome. Int J Radiat Oncol Biol Phys. 1997;39(3):711-9.

33. Tian YM, Xiao WW, Bai L, Liu XW, Zhao C, Lu TX, et al. Impact of primary tumor volume and location on the prognosis of patients with locally recurrent nasopharyngeal carcinoma. Chin J Cancer. 2015;34(6):247-53.

34. Guo R, Sun Y, Yu XL, Yin WJ, Li WF, Chen YY, et al. Is primary tumor volume still a prognostic factor in intensity modulated radiation therapy for nasopharyngeal carcinoma? Radiother Oncol. 2012;104(3):294-9. 
35. Tan T, Lim WT, Fong KW, Cheah SL, Soong YL, Ang MK, et al. Concurrent chemo-radiation with or without induction gemcitabine, carboplatin, and paclitaxel: a randomized, phase $2 / 3$ trial in locally advanced nasopharyngeal carcinoma. Int J Radiat Oncol Biol Phys. 2015;91(5):952-60

36. Sun X, Su S, Chen C, Han F, Zhao C, Xiao W, et al. Long-term outcomes of intensity-modulated radiotherapy for 868 patients with nasopharyngeal carcinoma: an analysis of survival and treatment toxicities. Radiother Oncol. 2014;110(3):398-403. doi:10.1016/j.radonc.2013.10.020.
37. Kong L, Zhang YW, Hu CS, Guo Y. Neoadjuvant chemotherapy followed by concurrent chemoradiation for locally advanced nasopharyngeal carcinoma. Chin J Cancer. 2010;29(5):551-5.

\section{Submit your next manuscript to BioMed Central and we will help you at every step:}

- We accept pre-submission inquiries

- Our selector tool helps you to find the most relevant journal

- We provide round the clock customer support

- Convenient online submission

- Thorough peer review

- Inclusion in PubMed and all major indexing services

- Maximum visibility for your research

Submit your manuscript at

www.biomedcentral.com/submit 